\title{
Re-Entry Aeroheating Analysis of Tile-Repair Augers for the Shuttle Orbiter
}

\author{
Ali R. Mazaheri, and William A. Wood ${ }^{\dagger}$
}

\begin{abstract}
Computational re-entry aerothermodynamic analysis of the Space Shuttle Orbiter's tile overlay repair (TOR) sub-assembly is presented. Entry aeroheating analyses are conducted to characterize the aerothermodynamic environment of the TOR and to provide necessary inputs for future TOR thermal and structural analyses. The TOR sub-assembly consists of a thin plate and several augers and spacers that serve as the TOR fasteners. For the computational analysis, the Langley Aerothermodynamic Upwind Relaxation Algorithm (LAURA) is used. A 5-species non-equilibrium chemistry model with a finite rate catalytic recombination model and a radiation equilibrium wall condition are used. It is assumed that wall properties are the same as reaction cured glass (RCG) properties with a surface emissivity of $\epsilon=0.89$. Surface heat transfer rates for the TOR and tile repair augers (TRA) are computed at a STS-107 trajectory point corresponding to Mach 18 free stream conditions. Computational results show that the average heating bump factor (BF), which is a ratio of local heat transfer rate to a design reference point located at the damage site, for the auger head alone is about 1.9. It is also shown that the average BF for the combined auger and washer heads is about 2.0 .
\end{abstract}

\section{Nomenclature}

\begin{tabular}{llll}
$H$ & Altitude, $[\mathrm{ft}]$ & \multicolumn{2}{l}{ Abbreviations } \\
$i, j, k$ & Grid points indices in & BF & Normalized heat transfer rate, Bump factor \\
& curvilinear computational coordinates & BPt & Body point \\
$k$ & Tile overlay thickness, $[$ inches $]$ & CAIB & Columbia accident investigation board \\
$M$ & Mach number & OML & Outer mold loop \\
$q_{w}$ & Surface heat transfer rate, $\left[B T U / f t^{2}-s e c\right]$ & RCG & Reaction cured glass \\
$T$ & Temperature, $[R]$ & SiC & Silicon carbide \\
$V$ & Velocity, $[\mathrm{ft} / \mathrm{sec}]$ & STS & Space transportation system \\
$x, y, z$ & Reference coordinate system & TOR & Tile overlay repair \\
$\alpha$ & Angle of attack, $[$ degrees $]$ & TPS & Thermal protection system \\
$\delta$ & Boundary layer thickness, $[$ inches $]$ & TRA & Tile repair auger \\
$\rho$ & Density, $\left[\right.$ slugs $\left./ \mathrm{ft}^{3}\right]$ & & \\
$\epsilon$ & Emissivity & &
\end{tabular}

\section{Introduction}

description of the process utilized to perform Orbiter tile damage assessment is reported by Campbell et Aal. ${ }^{3}$ In that report, individual aeroheating tools with nominal re-entry heating environment characterizations are emphasized, where Orbiter aeroheating environment is defined by nominal smooth body heating. Smooth body refers to an undamaged Thermal Protection System (TPS). Accordingly, critical damage to the TPS is characterized by three different damage scenarios. These scenarios are damage that 1) does not generate a catastrophic situation but may create a sequence of significant damage propagations which leads to an unsafe situation, 2) does not generate a catastrophic situation but minimal damage propagation may

*AMA, Inc./NASA LaRC, Aerothermodynamics Branch, AIAA Member; Ali.R.Mazaheri@nasa.gov

${ }^{\dagger}$ NASA Langley Research Center, Aerothermodynamics Branch, Senior lifetime AIAA Member. 
lead to an unsafe situation, 3) does generate an unsafe situation due to a severe damage. In this study, only the tile damage that is considered as a second category problem out of these scenarios is addressed. The current tile-repair option is a thin shell outer surface barrier, which is referred to as the Tile Overlay. This tile overlay is a thin Carbon Silicon Carbide plate that is placed on a damaged surface of the orbiter and attached with several screws and washers. These screws and washers are referred to as augers and spacers, respectively, in the present paper. Figure 1 shows the tile overlay, cavity insulation, gasket, and fasteners.

In this study, the effects of tile-repair augers on surface heat transfer rate are investigated. To minimize computational time, smooth body Orbiter CFD data was used as a baseline solution.

\section{Computational Modeling Strategy}

For the computational analysis, a 5species non-equilibrium chemistry model and a finite rate catalytic recombination model were used. Thin-layer Navier Stokes was solved with thermal equilibrium condition. Computational analyses were performed using the Langley Aerothermodynamic Upwind Relaxation Algorithm (LAURA) ${ }^{7,8}$ as a multispecies reacting viscous flow solver. Simulations were conducted at flight conditions corresponding to Mach 18 of the Space Shuttle Orbiter STS-107 trajectory. The exact free stream Mach number is 17.85 . A non-ionizing gas was considered as ionization does not occur until the temperature reaches about $16200 \mathrm{R}$. Based on the flow properties at a trajectory point given in Table 1, and the velocity-altitude map reported by Anderson ${ }^{2}$ and Koppenwallner, ${ }^{10}$ the air is not ionized at this point. A radiation equilibrium wall condition with reaction cured glass (RCG) properties and with surface emissivity of $\epsilon=0.89$ was assumed. An engineering approximation can be made for different emissivity and catalycity conditions by multiplying the emissivity ratio with the surface heating factor, BF; higher emissivity leads to a lower surface temperature. Computations were performed for only laminar flow conditions. The possibility that the boundary layer may be transitional and turbulent downstream of the protuberance is not considered here.

Table 1. Free Stream Flow Conditions.

\begin{tabular}{cccccc}
\hline $\mathrm{H}$ & $\mathrm{T}$ & $\mathrm{V}$ & $\mathrm{M}$ & $\rho \times 10^{6}$ & $\alpha$ \\
$f t$ & $R$ & $f t / s$ & - & slugs $/ \mathrm{ft}^{3}$ & degrees \\
196850 & 772.67 & 18164.34 & 17.85 & 0.5058 & 39.02 \\
\hline
\end{tabular}

The objective of the computational aeroheating analysis is to identify the effects of Tile Repair Augers (TRA) on the aerothermodynamic environment of the Tile Overlay Repair (TOR). Because of the hypersonic nature of the flow surrounding the TOR and relatively small thickness of the TOR and TRA flow disturbances are negligible in areas that are far from the TOR surface. Therefore, the existing solution of the smooth-body Shuttle Orbiter or smooth OML was used as a baseline solution, and the TOR was embedded using a grid morphing algorithm ${ }^{9}$ within the flow field of the computed solution. The local solution was then recomputed to account for the TOR and TRA protuberances.

The process begins by locating the damage site on the Orbiter surface. The damage location considered in this study is known as Body Point (BPt) 1800. Flow properties at this reference point are given in Table 2. The TOR surface geometry was generated and projected on the smooth OML surface after the TOR was aligned with a desired orientation. Schematic of the damage site with the TOR orientation is shown in Figure 2. The TOR surface size is 15 inches by 25 inches with 3.5-inch rounded corner radii ${ }^{\mathrm{a}}$. The TOR thickness is 0.15 inches. The TOR and the surrounding area are modeled with a 56 block structured grid. The resulting surface grid is shown in Figure 2. The computational domain and boundary conditions used are shown in Figure 3. In this analysis the overlay plate central bulge that is due primarily to the presence

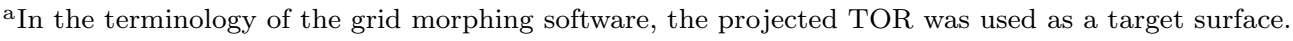


of a gasket underneath the TOR is not included. The gasket position is schematically shown in Figure 1.

Table 2. Flow properties at BPt 1800 and its location in Shuttle coordinates $\dagger$.

\begin{tabular}{ccccc}
\hline $\mathrm{x}$ & $\mathrm{y}$ & $\mathrm{z}$ & $\delta$ & $q_{w}$ \\
inches & inches & inches & inches & $B T U / f t^{2}-$ sec \\
1265 & 0 & 261 & 2.02 & 4.96 \\
\hline
\end{tabular}

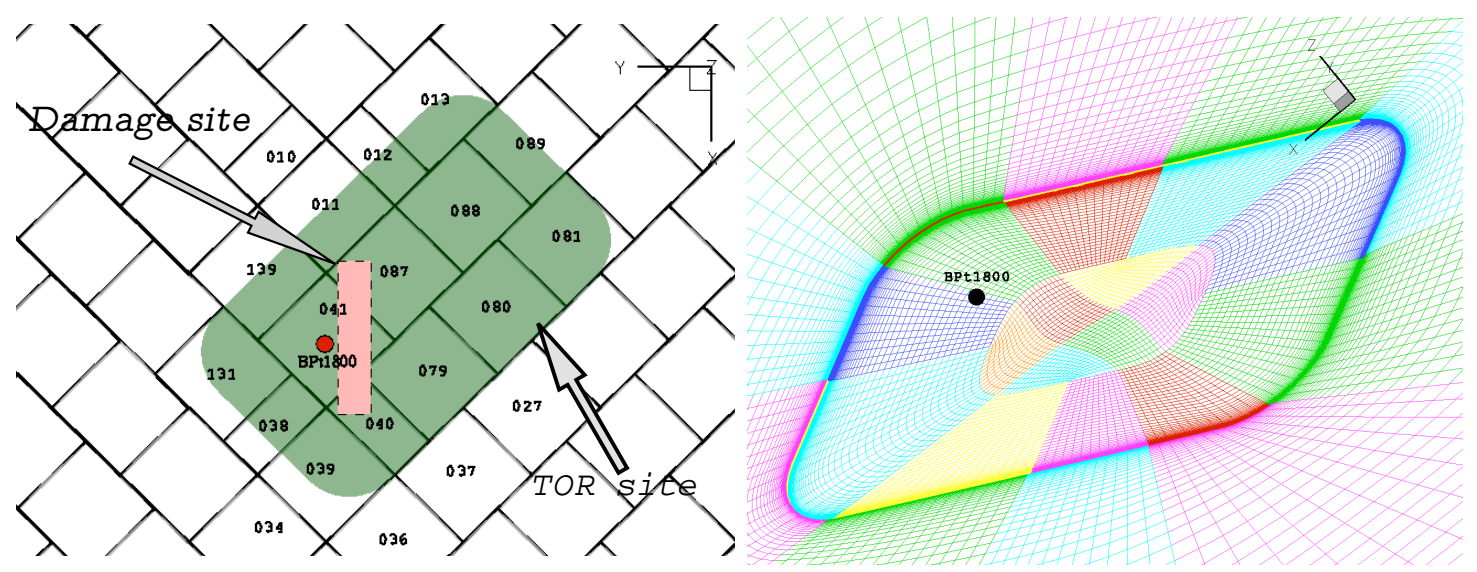

Figure 2. left: Schematic of the damage site and TOR relative to Atlantis tile layout. (View from outside of the Orbiter, with its nose toward top of the page.); right: Schematic of the tile overlay with its structured grid.

Similar steps were taken to model the TRA. The tile repair auger was modeled as a circular protuberance with diameter of 0.85 inches and a height above surface of 0.0552 inches. Table 3 gives the coordinates of the two augers that were analyzed. The TRA locations on the TOR are shown in Figure 4. Top and side views of the actual and the simplified auger and spacer geometries on the TOR are schematically shown in Figure 5. In the computational analyses of the TRA the simplified geometry was used.

Table 3. Coordinates of the TRA at their centers.

\begin{tabular}{ccc}
\hline $\mathrm{x}$ & $\mathrm{y}$ & $\mathrm{z}$ \\
inches & inches & inches \\
1250.58 & -9.48 & 260.44 \\
1251.85 & -11.04 & 260.43 \\
\hline
\end{tabular}

For parametric analysis, augers were modeled with several grid topologies. These grids are schmetically shown in Figure 6. In Grids a and b, TRA was created with four grid-blocks but with different grid distributions and resolutions for the auger head area. Grid c is similar to Grid a but with eight grid-blocks. Grid $d$ is more refined than the first three grids, and was created with fourteen grid-blocks. Also, a TRA model with much larger domain than those shown in Figure 6 was modeled to investigate sufficiency of the computational domain in computational aeroheating analysis. For the larger domain case, a computer program was developed to merge a morphed auger with the morphed TOR, considering just the most upstream auger. The resulting larger domain includes both the TOR computational domain shown in Figure 2 and the TRA domain shown in Figure 6. Figure 7 shows a multi-block surface grid for this larger domain. In this case, grid resolution (b) of Figure 6 was adapted for the spacer and auger. A total of 119 blocks was generated for this case.

A tandem TRA was also modeled to investigate flow channeling. For this case, the two augers shown in Figure 4 were separately placed on the TOR with the same method that was described above. The grid-morphed augers were then merged together using a developed merging program (an in-house program). The boundaries of the tandem auger and the merged multi-block structured grids are shown in Figure 8. 


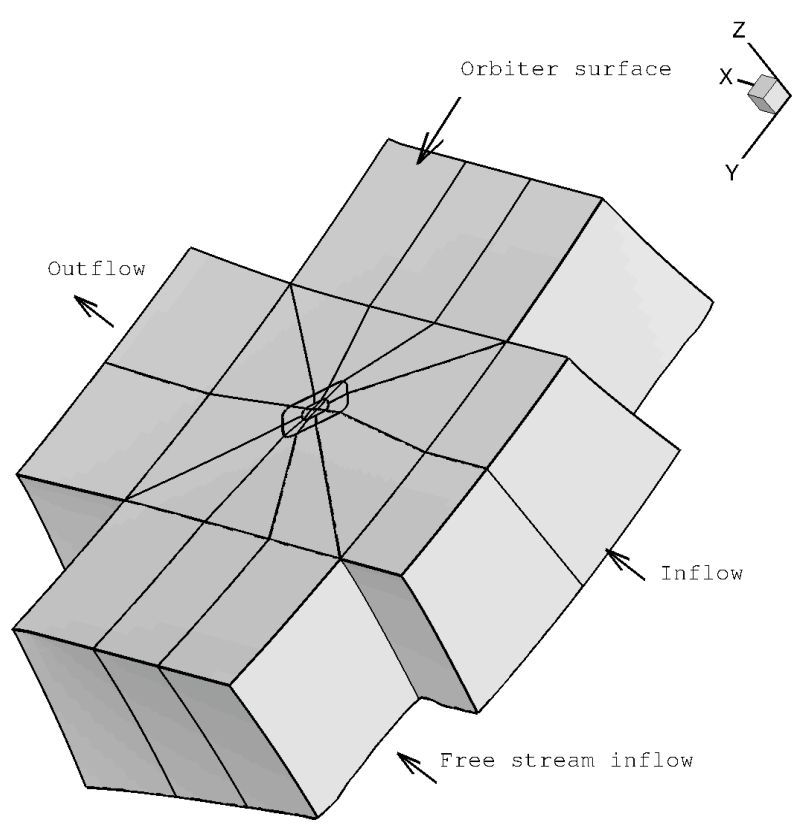

Figure 3. Schematic of the computational domain and boundary conditions used for aerothermodynamic analysis of the TOR. (The view is looking from the inside of the Orbiter structure out into the flowfield.)

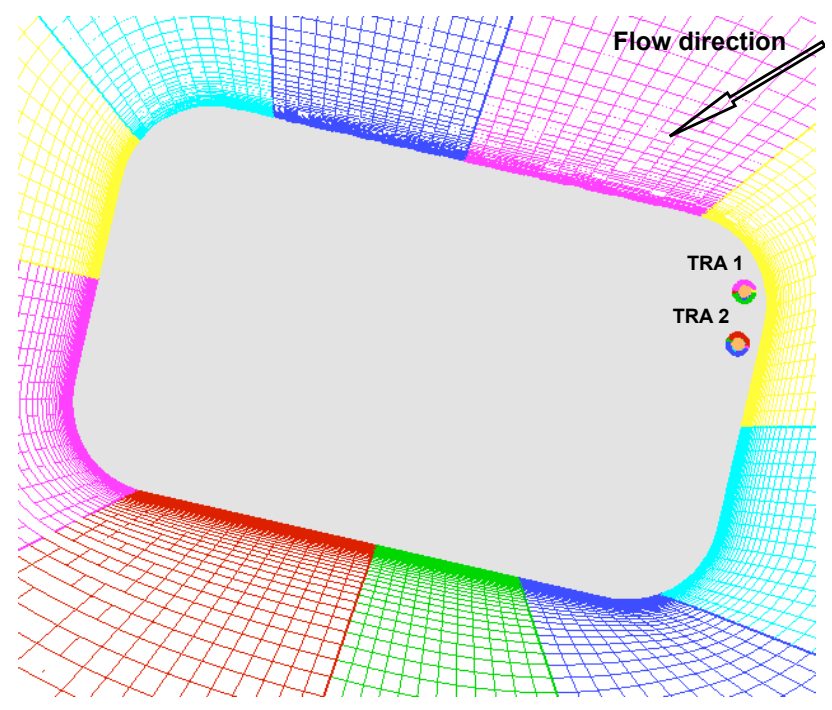

Figure 4. Schematic of the TRA locations on the TOR (Positioning is in the upstream corner.) 

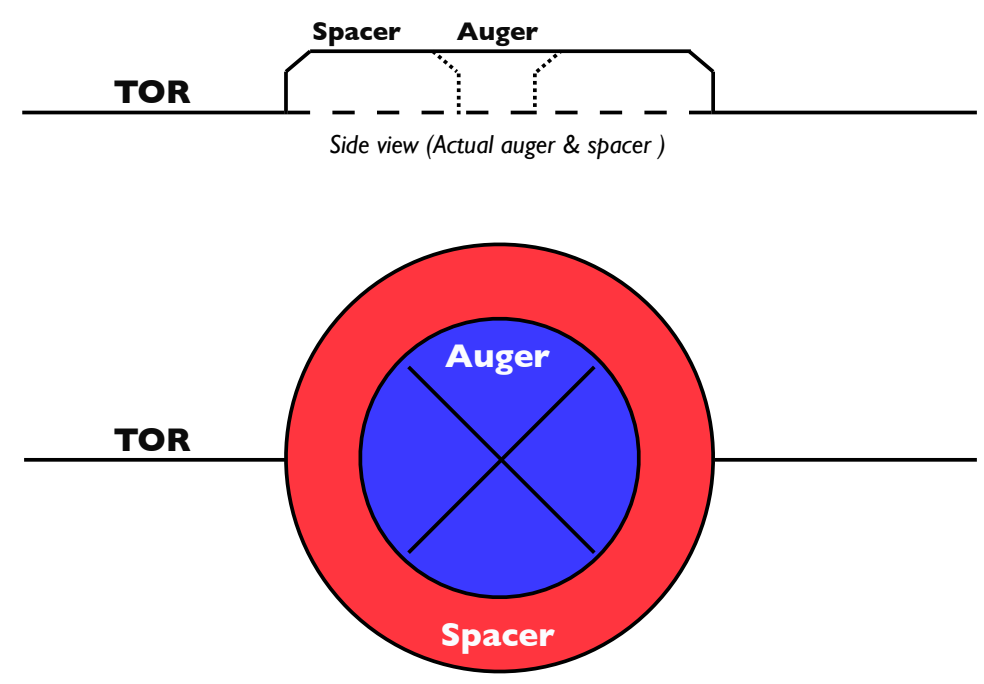

Top view

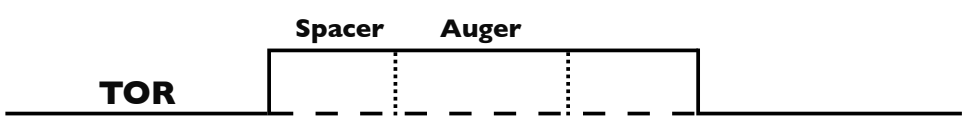

Side view (Simplified auger \& spacer)

Figure 5. Schematic of actual and simplified TRA geometries on the TOR.

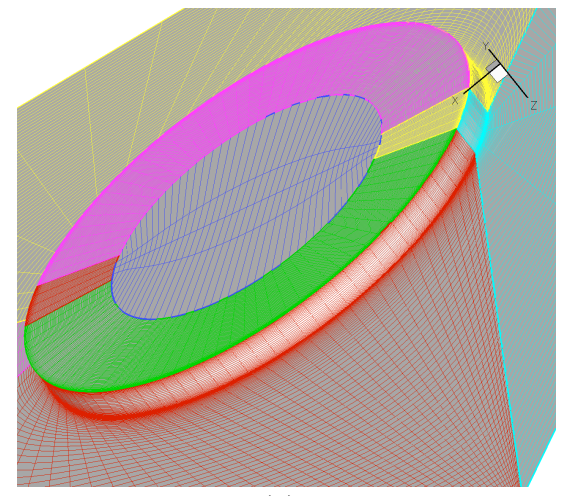

(a)

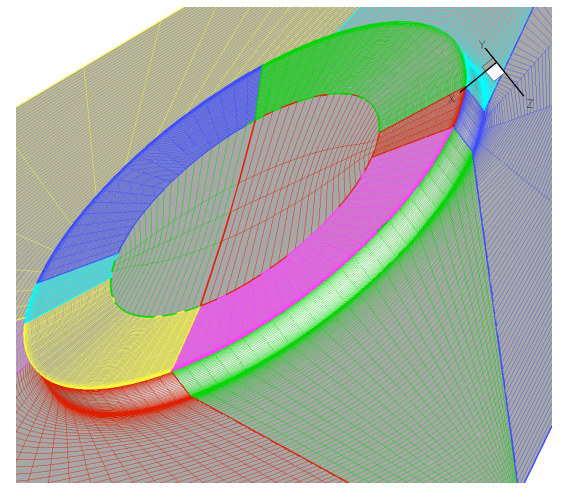

(c)

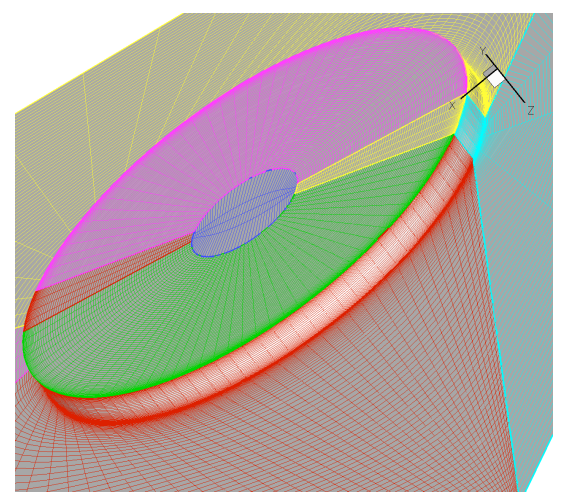

(b)

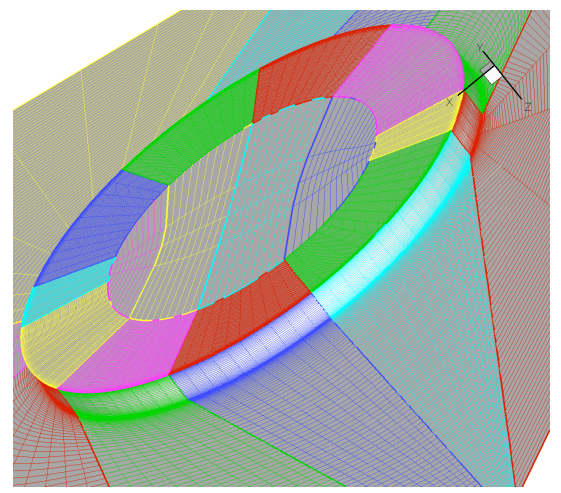

(d)

Figure 6. Computational grids for the auger and spacer heads with different resolutions; a) five grid-blocks, b) five grid-blocks, c) eight grid-blocks, d) fourteen grid-blocks. 


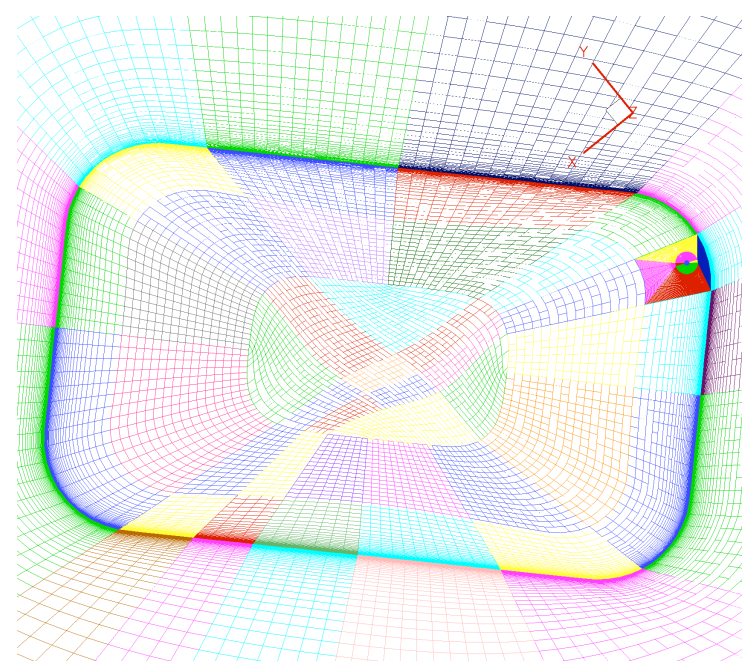

Figure 7. Surface grid used for the spacer aeroheating analysis using larger computational domain.

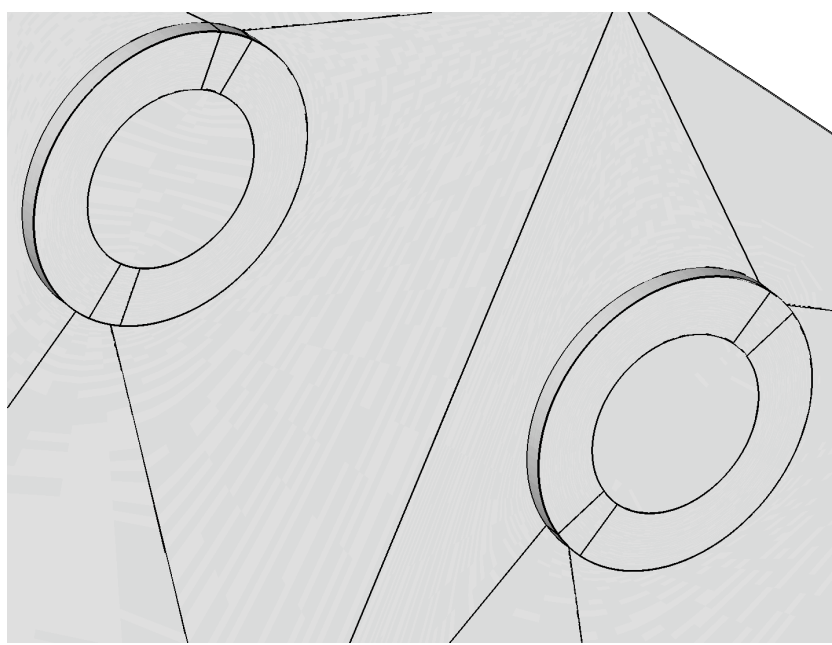

(a)

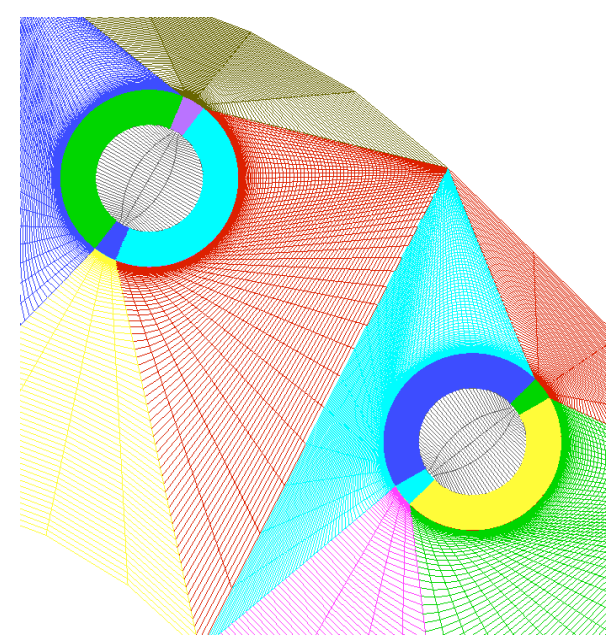

(b)

Figure 8. Schematics of a) geometries and boundaries of the tandem TRA, and b) the tandem multi-block structured grid. 


\section{Computational Results}

Entry aeroheating analysis of the TOR and TRA are performed at the BPt 1800 design reference point. Free stream flight conditions were taken from the Mach 18 STS-107 trajectory point listed in Table 1. Effects of the protuberances on the local heat transfer rates were studied. Local heat transfer effects were characterized by dividing the local heat transfer rate by that of the smooth OML BPt 1800. This ratio is referred to as the Bump Factor (BF). Figure 9 shows the surface heat flux relative to its value at the BPt 1800 on the smooth OML surface.

\section{A. Tile Overlay Repair (TOR)}

Bump factor variation on the TOR surface without the augers is shown in Figure 10. This figure shows approximately 2.5 times higher surface heat transfer rate on the upstream edge of the TOR when compared with that of BPt 1800. A relatively uniform heating BF is computed for the rest of the overlay in which the $\mathrm{BF}$ is slightly higher than unity. The result of a more detailed analysis of overlay surface $\mathrm{BF}$ variation is also shown in this figure. Figure 10b shows tile surface BF with distance measured from the most upstream corner and along the flow direction. This figure shows that the BF decreases sharply from about 2.4 to about 1.2 as flow moves away from the TOR corner. An inset of the figure shows the surface $\mathrm{BF}$ variation excluding the edges

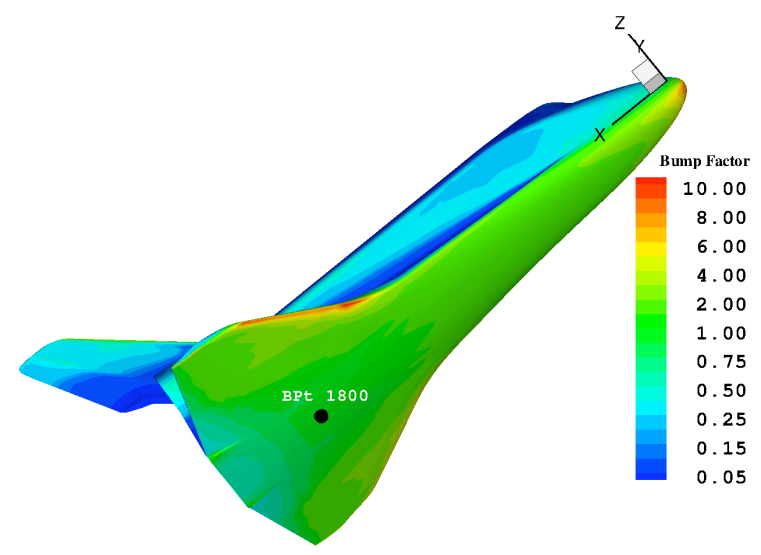

Figure 9. Contour plot of smooth OML surface heating BF at $M a c h=18$ and $\alpha=39^{\circ}$. with stretched y-axis. near the upstream TOR edge. This close-up figure shows a minimum BF of about 1.0 at a distance of about 10 inches from the upstream TOR edge. Figure 10c shows the tile overlay surface bump factor across its 25 inch length as shown with the arrow. The BF decreases quickly from its value of about 1.5 at the tile edge to about 1.0, and continues to decrease until it reaches about $80 \%$ of its width and then increases to about 1.20 as it gets to the downstream edge of the tile overlay.

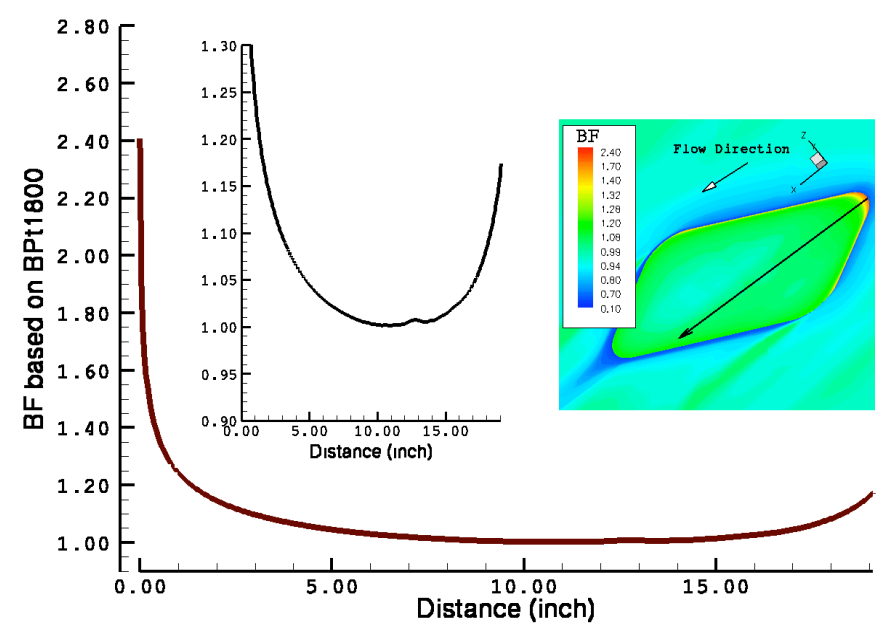

(a)

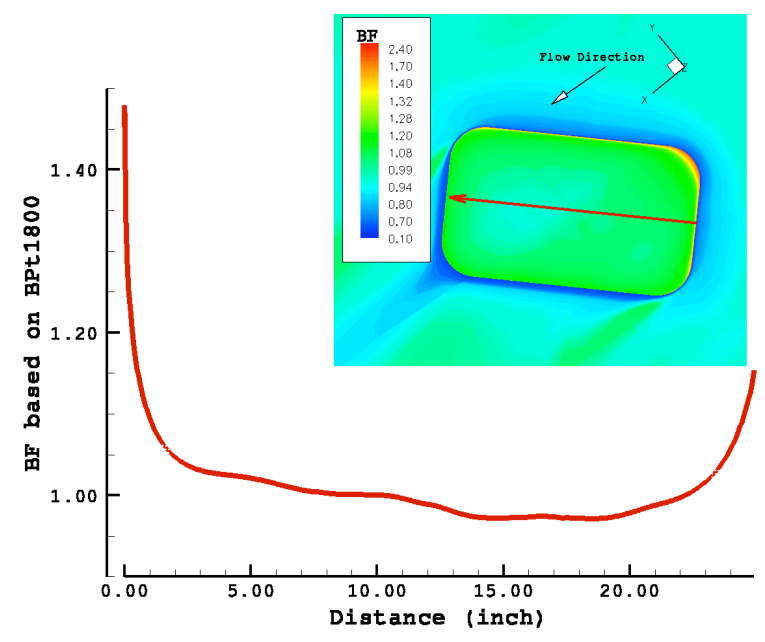

(b)

Figure 10. Variations of the tile overlay surface BF a) with distance from the upstream corner of the overlay along the flow direction, and b) with distance across the tile overlay. 


\section{B. Tile Repair Augers (TRA)}

Several augers are used to install the tile overlay repair to the Space Shuttle surface. Due to higher conductivity of these augers and spacers than the TOR, heat may conduct to the layer underneath the tiles, leading to an increase in temperature at the tile bond line. The augers that are most critical to aeroheating analysis are those that are located near the TOR leading edge, which has the highest BF (see Figure 10.) These augers are considered for TRA areohermodynamic analysis.

For grid sensitivity analysis, computational re-entry aeroheating analyses were performed for the TRA with the grids shown in Figure 6. The TRA surface heating BF are shown in Figure 11. The computed results are similar using the different grids. Figure 11 shows a BF of about 3.0 at the edges of the spacer. The actual spacer geometry was approximated as a protuberance with sharp edges as opposed to rounded edges. Thus, the edge BF does not reflect a true value.

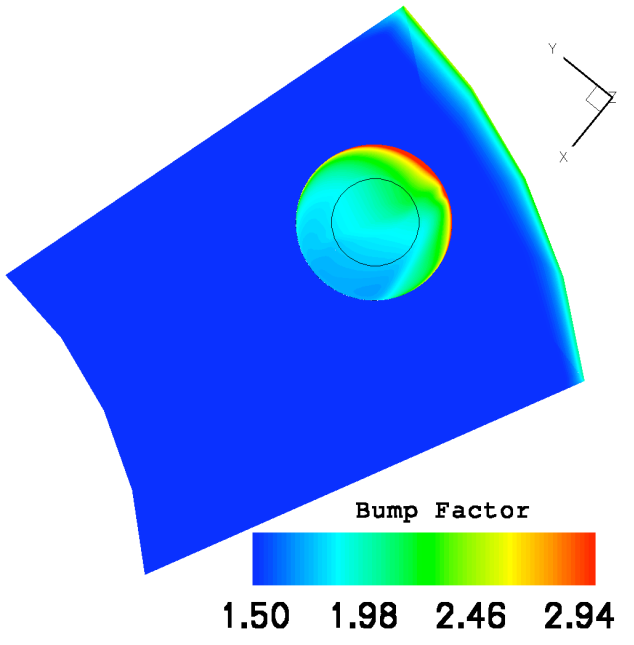

(a)

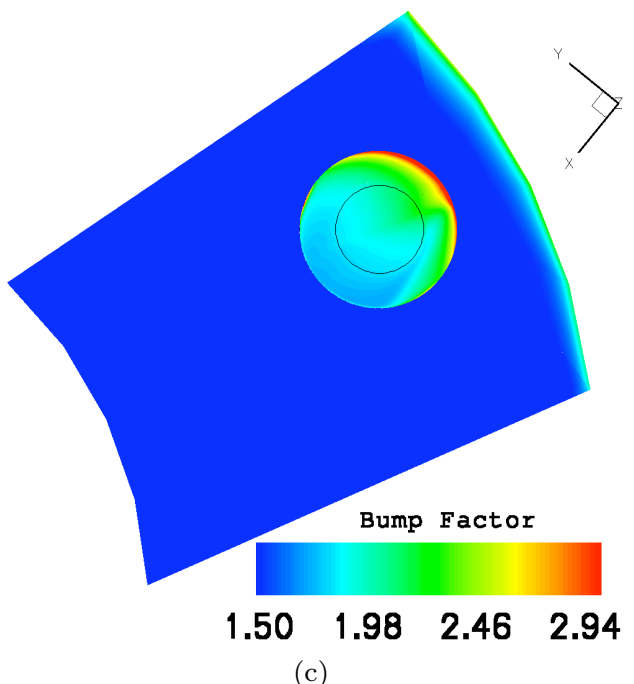

(c)

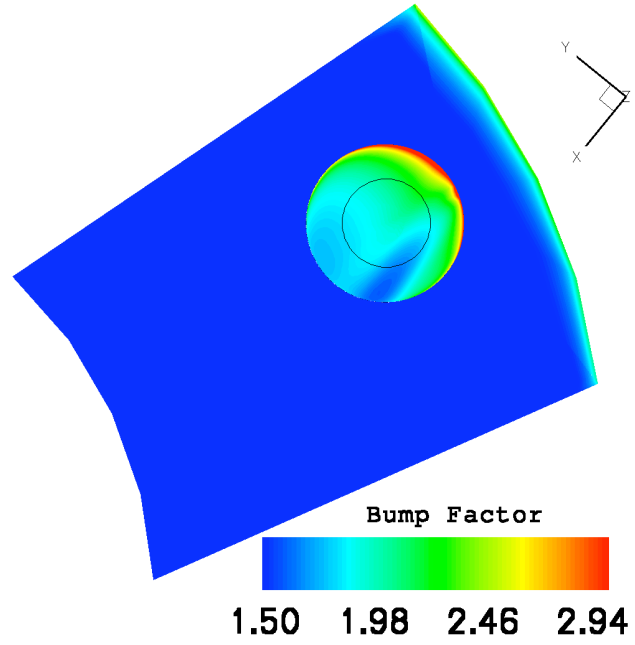

(b)

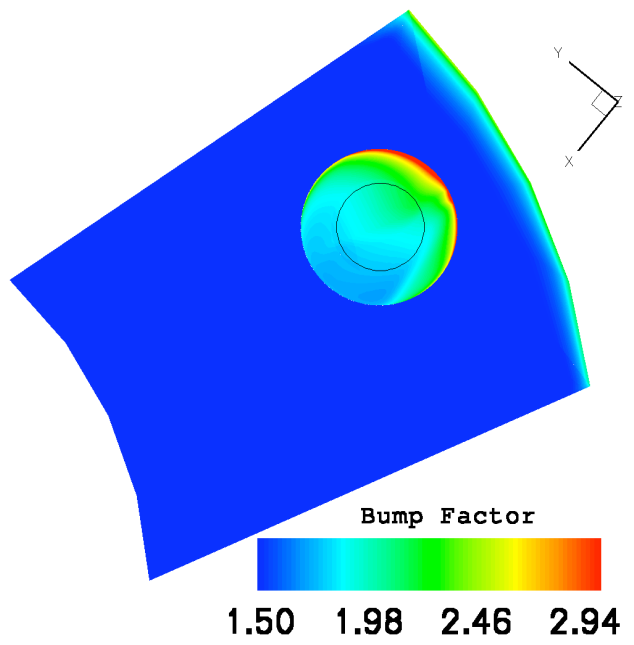

(d)

Figure 11. Contour variations of heating BF with grids (a), (b), (c), and (d) of Figure 6 at Mach 18 free stream conditions.

To estimate the total heat flux conducted through the auger shaft, BF values were integrated over the top surface area of TRA. These results are presented in Table 4, which shows an overall BF of about 1.9 for the auger head only and about 2.0 for auger and spacer top surfaces considered as a single unit. The bump factors are within 0.02 for all four different grid resolutions.

A more detailed surface BF distribution on auger and spacer heads is shown in Figure 12. This figure 
Table 4. Normalized heat transfer rate, BF, for auger and spacer surfaces with different grid resolutions.

\begin{tabular}{cccc}
\hline & & Average Bump Factor (BF) with respect to BPt 1800 \\
& & Auger Surface & Auger + SpACER Surfaces \\
& a & 1.91 & 2.02 \\
Grid Resolution & b & 1.93 & 2.02 \\
& c & 1.93 & 2.02 \\
& d & 1.91 & 2.02 \\
\hline
\end{tabular}

shows variations of the surface BF with two distances; one from the upstream edge of the TOR along the flow direction (shown with longer arrow) and the other perpendicular to the flow direction and across the spacer (shown with shorter arrow). The distance between the edges of the overlay and the spacer is marked upstream with negative values on the $x$ axis. The second part of the graph belongs to the auger and spacer heads. Part of the graph that corresponds to the auger head is also marked for clarification. Figure 12 shows that the BF decreases from about 2.4 at the upstream edge of the overlay to about 0.5 just upstream of the spacer edge. As the gas flow sees an immediate increase in surface height, the surface heat transfer jumps to a maximum value of $\mathrm{BF}=3.9$ at the edge of the spacer. The $\mathrm{BF}$ then dissipates as the flow moves toward the other side of the spacer. The auger head and spacer surface BF on a plane perpendicular to the flow direction is also shown in Figure 12. Bump factor variation is shown with a dark line in this figure, which shows a relatively uniform surface heat flux with nominal BF value of about 2.0 .

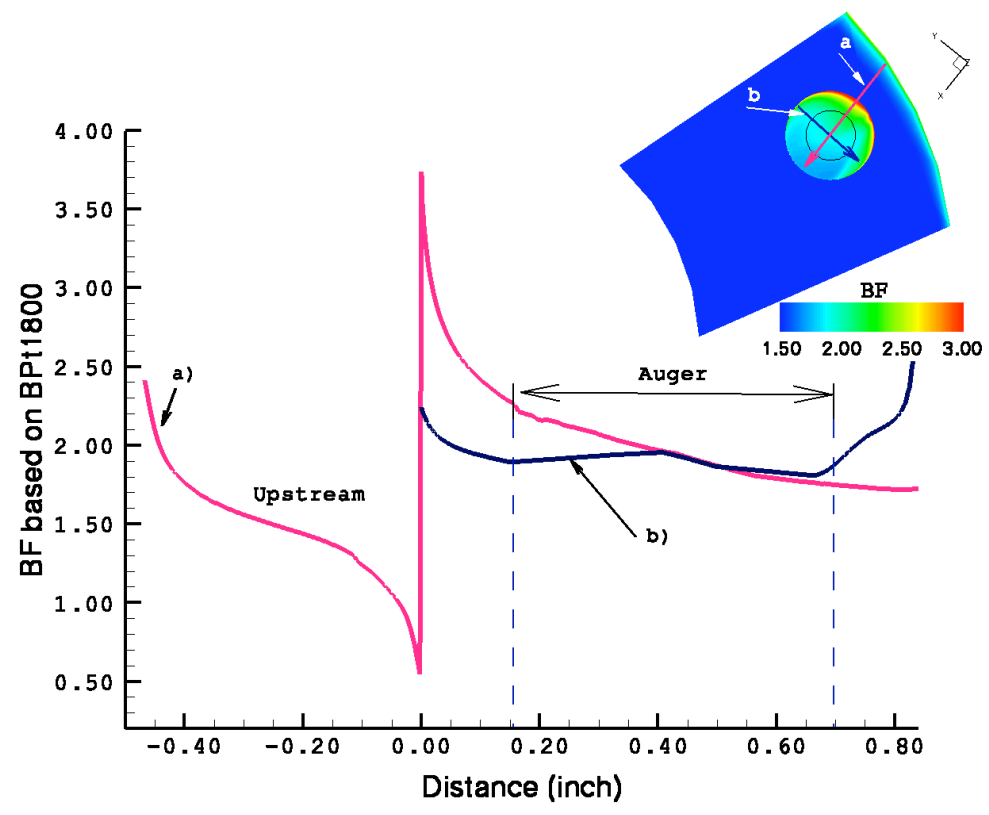

Figure 12. Variations of the auger and spacer surfaces BF with distances a) from the upstream edge of the tile overlay, along the flow direction, and b) across the spacer and auger, perpendicular to the flow direction. 0.00 marks the outer edge of the top of the spacer.

To verify that the size of the computational domain that was used is sufficient in the aeroheating analysis, a larger computational domain (see Figure 7) was developed and the results were compared with the previous models. Figure 13 shows the computed aeroheating results of BF for the spacer, auger, and TOR surfaces. In this figure, $\mathrm{BF}$ variation with distance that is measured from the upstream corner of the TOR and along the flow direction is shown. Figure 13 shows that the BF quickly drops from about 2.2 on the TOR corner to about 0.8 just before the spacer. This is because of flow separations in front of the spacer. The BF increases instantly to about 3.3 at the most upstream corner of the spacer. This BF then decreases to a minimum value of about 2.0 on the most downstream location of the spacer before it falls to about 0.4 on the TOR surface just downstream of the spacer. The BF jumps quickly to about 1.2 afterward, and monotonically 
increases to about 1.5 on the other side of the TOR.

In order to compare the results, average surface bump factors on the spacer and auger surfaces were evaluated. The average surface BF for the combined auger and spacer surfaces is 2.05 , close to the reported value of 2.02 in Table 4 using a smaller computational domain. Computational aerothermodynamic analyses of the spacer and auger on the TOR using a larger domain provide a larger view of the tile overlay surface heat fluxes.

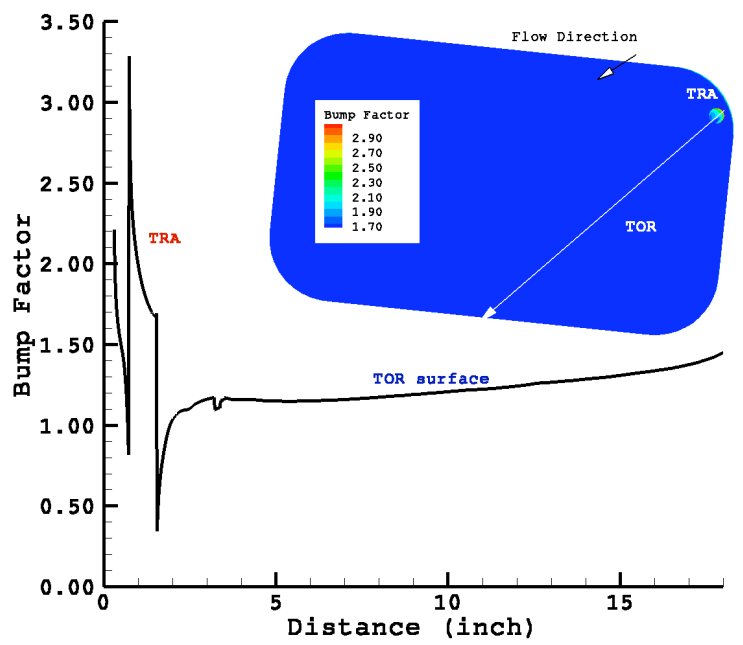

Figure 13. Surface BF variations on the TRA and TOR surfaces with distance from the leading edge of the TOR along the flow direction.

Effects of tandem augers and spacers on the aeroheating were also investigated. The location of the tandem auger configuration is schematically shown in Figure 8. In this case, grid (a) of Figure 6 was adapted for each one of the augers and spacers. Both spacers and augers were included in the aeroheating calculations; to allow for possible interference effects the single spacer solution was not reused during this analysis. Figure 14 shows BF variation for the tandem augers. It is shown that a large portion of the tandem surface is exposed to high temperature gas flow; no noticeable effects of the upstream spacer (TRA-1) on the downstream spacer (TRA-2) surface heat transfer rate was observed. Also, flow-channeling did not occur between these spacers. Asymmetric heat transfer rate on the TRA-2 indicates the presence of flow channeling. Similar BF pattern on the both TRAs shows that a negligible effect of the upstream spacer (TRA-1) on the downstream spacer (TRA-2.)

Similar to the graph shown in Figure 12, a line-cut through the downstream spacer and auger is shown in Figure 14 for comparison. This figure shows that heat transfer rate remains unchanged, due to adding the second TRA, with BF of about 1.90 for the auger head only. However, the upstream spacer surface BF is increased slightly with the combined auger and spacer BF of about 2.07. Note that based on the aeroheating results of the individual auger and spacer on the tile overlay, BF is about 2.02. Figure 14b shows a decrease in surface BF from the leading edge of the TOR and a sudden increase in its value at the upstream edge of the spacer to a maximum value of about 3.0. This high surface heat transfer rate drops quickly and reaches a minimum value of about 1.6 downstream of the spacer. These results are summarized in Table 5. This table indicates that the downstream auger surface BF is about 1.77, which is less than that for the upstream one. The combined auger and spacer BF for the downstream one is about 1.91. A comparison was made with the above study where only the upstream auger was modeled. Table 5 shows that the auger BF located on the most upstream edge of the tile overlay is not affected by the downstream spacer and auger.

\section{Conclusions}

In this investigation re-entry aeroheating analyses were conducted for the Space Shuttle Orbiter. Aerothermodynamic properties of a specific Tile Overlay Repair (TOR), including its spacers and augers were numerically analyzed using the Langley Aerothermodynamics Upwind Relaxation Algorithm (LAURA). Flow conditions were extracted from a STS-107 trajectory point corresponding to a free stream Mach number of 


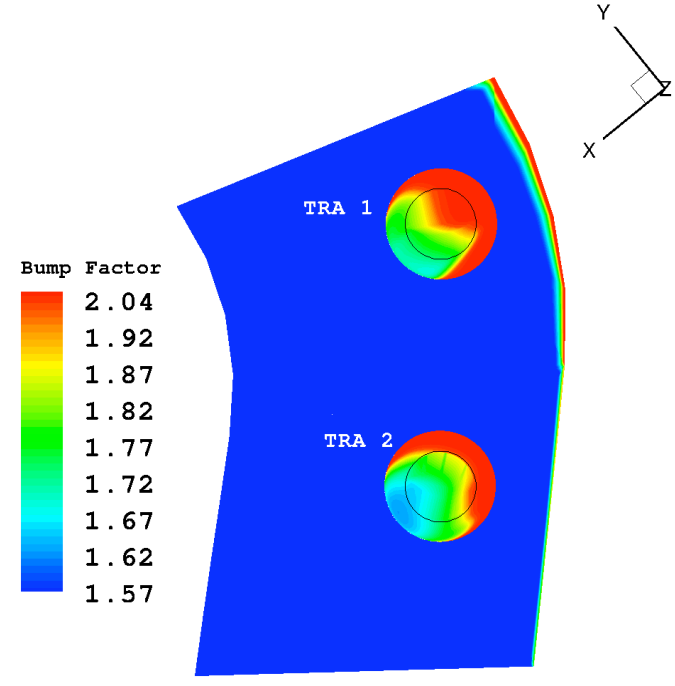

(a)

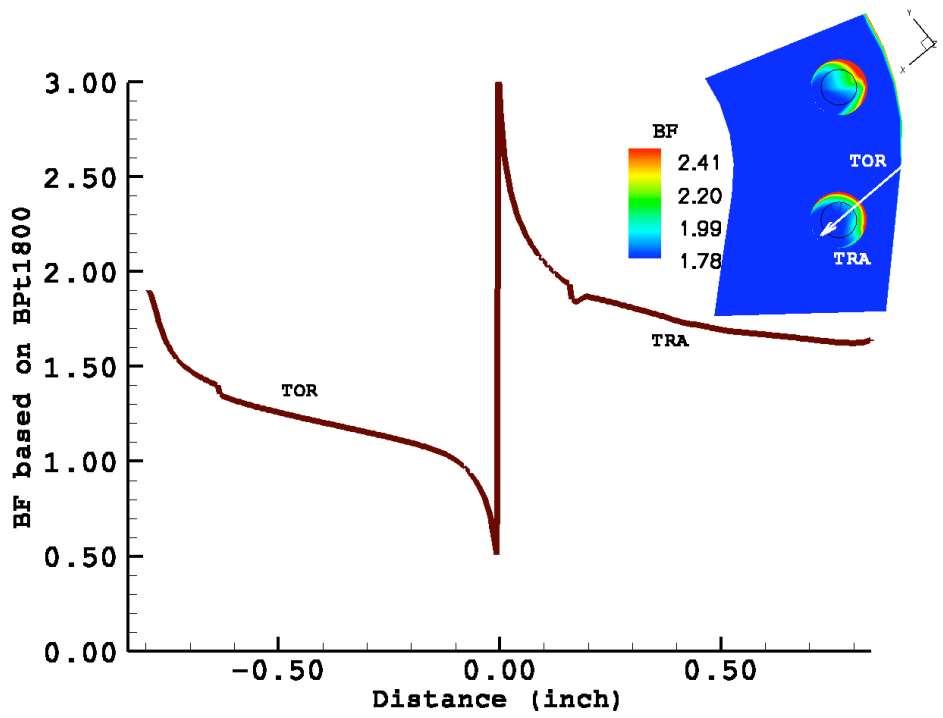

(b)

Figure 14. Variations of surface heating BF for the tandem auger and spacer surfaces at Mach 18 free stream conditions.

Table 5. Tabulated data of heating BF for auger and spacer surfaces for different modeling types.

\section{Modeling Type}

Tandem

Individual (from Table 4)

\section{Average Bump Factor (BF)}

Auger Surface Auger + Spacer Surfaces

$1.91 \quad 2.07$

$1.77-1.91$

18. Chemical non-equilibrium, thermal equilibrium, non-ionizing five species gas was assumed, and surface heat transfer rate distributions were computed. The results are summarized as following:

- The average aeroheating analyses are insensitive to the grid variations studied in this investigation.

- In modeling the individual auger on the TOR, the average heating BF is about 1.9. The combined auger and spacer top surface BF is about 2.02 .

- In analysis of the tandem auger, the upstream spacer did not noticeably interfere with the aeroheating properties of the downstream auger. The average downstream auger surface BF is about 1.77, while the combined auger and spacer surface BF is about 1.91 .

\section{Acknowledgments}

The work of the first author was performed under the contract number NNL06AC49T to the NASA Langley research center.

\section{References}

${ }^{1}$ Aero/Aerothermal/Thermal/Structures team Final Report; in support of the CAIB, NTST-37398, 2003.

${ }^{2}$ Anderson, J.D., High Temperature Gas Dynamics, McGraw-Hill Book Co, 1985.

${ }^{3}$ Campbel, c.H., Anderson, B., Bourland, G., Bouslog, S., Cassady, A., Horvath, T.J., Berry, S.A., Gnoffo, P.A., Wood, W.A., Reuther, J.J., Driver, D.M., Chao, D.C., Hyatt, J., and Picetti, D., "Orbiter Return to Flight Aeroheating," 9 AIAA/ASME Joint Thermophysics and Heat Transfer Conference, San Francisco, CA, June 5-8, 2006.

${ }^{4}$ Columbia Accident Investigation Board Final report, Vols I-IV, URL: htttp://caib.nasa.gov, October, 2003. 
${ }^{5}$ Everhart, J.L., Alter, S.J., Merski, N.R., Wood, W.A., and Prabhu, R.K., "Pressure Gradient Effects on Hypersonic Cavity Flow Heating," AIAA 2006-0185, 2006.

${ }^{6}$ Everhart, J.L., Bey, K.S, Wood, W.A., and Pulsoneti, M.V., "Experimental and Computational Investigation of Cavity Flows Simulating damage to Thermal Protection System Tiles," $21^{\text {st }}$ JANNAF Airbreathing Propulsion Subcommittee, Colorado Springs, CO, December 1-5, 2003.

${ }^{7}$ Gnoffo, P.A., "An Upwind-Biased, Point-Implicit Relaxation Algorithm for Viscous, Compressible Perfect-Gas Flows," NASA TP2953, February 1990.

${ }^{8}$ Gnoffo, P.A., Gupta, R.N., and Shinn, J.L., "Conservation Equations and Physical Models for Hypersonic Air Flows in Thermal and Chemical Nonequilibrium," NASA TP2867, February 1989.

${ }^{9}$ Gnoffo, P.A., "Grid Morphing User Manual-Version 1.0", NASA LaRC, November, 2005.

${ }^{10}$ Koppenwallner, G., "Fundamentals of Hypersonics: Aerodynamics and Heat Transfer, " in the short course entitled Hypersonic Aerothermodynamics, presented at the Von Karman Institute for Fluid Dynamics, Rhose Saint Genese, Belgium, February 1984. 